\title{
AN IMPROVED DARK-ADAPTOMETER *
}

\author{
BY \\ WALTER KOCH
}

DEPARTMENT OF HYGIENE, HEBREW UNIVERSITY, JERUSALEM

SOME time ago an instrument was described in this Journal intended for testing dark-adaptation in normal and sub-normal patients. However, when it was tried to investigate gross disturbances (e.g., retinitis pigmentosa), the need was felt for a wider range of readings. When re-designing the apparatus provisions were also made to change the colour of the test-light if desired in order to make the instrument more versatile.

As the general outlay of the apparatus has remained the same except for two relevant items, the reader is referred to the previous paper. In the former design (see previous paper, p. 236, drawing I, "9") a blue glass plate was inserted into the path of rays; as this glass plate could not be easily removed, the light of the stimulus was unvarying blue. The same drawing also showed (see " $11^{\prime \prime}$ ) how the intensity of the test-light. was being reduced by openings of different size drilled into a metal-strip. A limit of this method was given by the fact that a very fine hole is rather difficult to drill, to keep clean, and to center.

In the present design the aforementioned limitations have been overcome as follows :

(a) Changing the colour of light: A double-walled, rotating disc (see 9A in this paper) replaces the blue filter (9) of the former design. This disc is similar to that used in ophthalmoscopes for changing lenses, and has six openings. Into five of them filters of different colour can be inserted; the sixth hole remains empty, thus passing uncoloured light when brought into position. In the photo the empty opening (6) is shown centered.

(b) Altering the intensity of light: A disc (see 11A in this paper), similar to the colour-filter disc (9A) takes the place of the metal strip (11) used in the previous design. The six openings in this disc are all of equal size and accomodate six grey filters of different transmission. These neutral screens may be obtained from Messrs. Ilford but if less accuracy is needed they can be prepared quite easily by exposing and developing photographic film. Their density* is determined by photometry. As very dark screens can be tested only with

* Received for publication, February 6, 1947.

* Density means $\log _{10}\left(\frac{I_{0}}{I}\right)$, where $I_{0}$ stands for incident light and $I$ for transmitted light; thus a filter transmitting 10 per cent. of the full (100 per cent.) incident light has a density of : $\log 100-\log 10$, or : $2-1=1$. 
difficulty, several more transparent neutral filters can be combined as to give the required density. As done for the disc carrying the coloured filters, the openings are marked from " 1 " to " 6 ". In the photo grey screen number three (with a density of 0.40 ) has been brought into position. The full range of densities is shown in table I :

\section{TABLE I}

\begin{tabular}{|c|c|c|c|c|c|}
\hline Number of opening: & $" 1 "$ & $" 2 "$ & $" 3 "$ & $" 4 "$ & $" 5^{\prime \prime}$ \\
\hline Density : & $2: 48$ & 1.49 & 0.40 & 5.78 & 4.63 \\
\hline
\end{tabular}

In order to determine the relative densities of our screens, one of them, e.g. " 1 " is assumed to be zero. Thus the density of screen two becomes: $1 \cdot 49-2 \cdot 48=-0 \cdot 99$, the density of screen three: $0.40-2.48=-2.08$ etc. Table II lists these values :

TABLIE II

$\begin{array}{lcccccc}\text { Number of opening: } & \text { " } 1 \text { " } & \text { "2" } & \text { "3" } & \text { "4" } & \text { "5" } & \text { "6" } \\ \text { Density - } 2.48: & 0 & -0.99 & -2.08 & +3.30 & +2.15 & +1.12\end{array}$

The calibration proper is performed without colour filter, but with grey screen one in position; a light-source calibrated by the National Physical Laboratory, Teddington, serves as a standard. By stopping down the diaphragm of the standard and of the adaptometer simultaneously, a calibration curve is obtained. Though these figures could be directly entered on the cardboard attached to the face of our shutter, it is more convenient to make a correction for blue light, as this colour is preferably used in dark adaptation tests. In the uncoloured light of our lamp the blue filter itself has a density of 2.37 ; thus $2 \cdot 37$ has to be deducted from the values obtained in the calibration chart. The corrected figures are now entered on the cardboard: The scale (as shown in the photo) reads directly logarithmic units of millimicrolamberts for blue light, if grey screen one is in position. Shifting over to grey screen two which is by 0.99 units more transparent (see Table II) means adding 0.99 to the reading of the diaphragm scale. Similarly; as grey screen six is by $1 \cdot 12$ units more opaque than screen one, it calls for a deduction of 1.12. Using white light instead of blue light, $2 \cdot 37$ has to be added (see above).

It will, however, be noticed that no arithmetic at all is involved when testing final thresholds in normal or slightly subnormal cases if blue light is used. For screen one (relative density zero) does not call for a correction. The neighbouring screen (two) which covers most of the sub-normal cases needs simply adding one (or more exactly $0 \cdot 99$ ). 


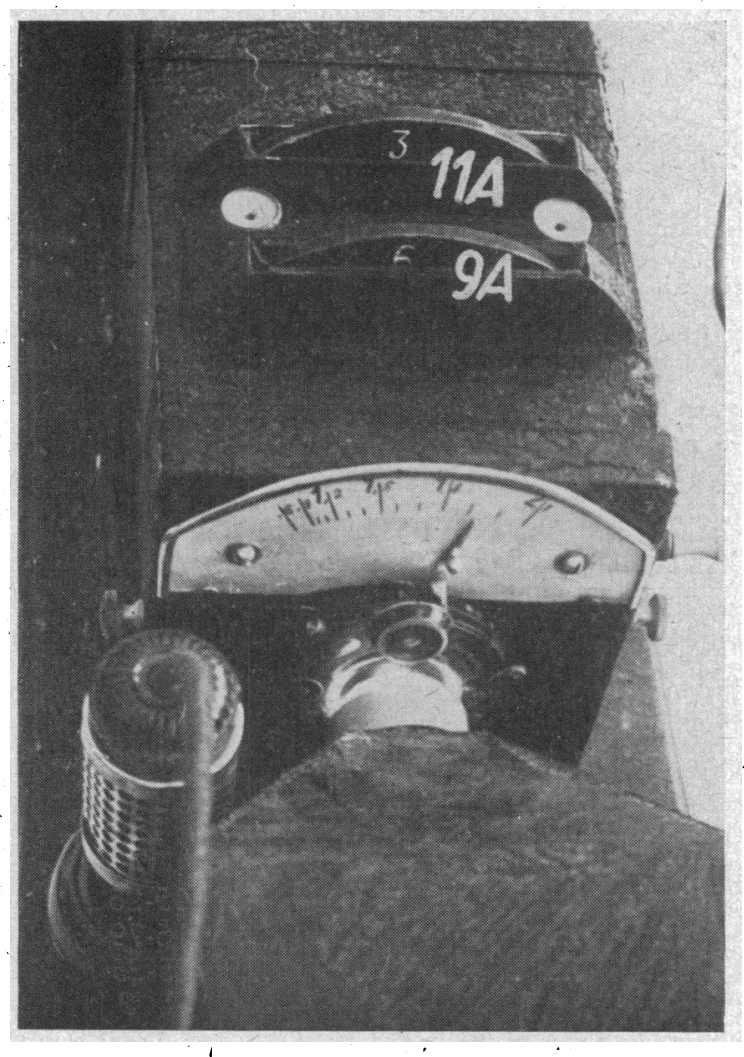

Thus the threshold shown in the photo has to be read off as : $1.9^{\circ}$ (indicated on the scale of the diaphragm), plus $2 \cdot 37 \cdot\left({ }^{\prime \prime} 6\right.$ " on the colour-filter disc indicating white light), plus $2 \cdot 08$ (due to grey screen " 3 "), the total value being $6.35 \mathrm{log}$ units of millimicrolamberts.

\section{Summary}

A dark-adaptometer is described which covers a wide range of intensities of light stimuli and allows the use of different colours.

\section{REFERENCE}

Kосн, W.-A new instrument for dark adaptation tests, Brit. Jl. Ophthal., May, 1945 , p. 234. 